tablishes the well-known caprice of the CASE 1.-L. A., ætat. 34, a strong musdisease in a most extraordinary manner. cular man, of temperate habits, living in a The caprice alluded to has been hitberto very low and confined part of the town, was chiefly manifested in its most irregular and seized early in the morning of the 20th unaccountable movements over large tracts July, with severe purging and nausea, of country, as India ; in this case, however, which continued very frequent, until five it would almost appear that it possesses a p.m., when $I$ first saw him. He attributed kind of voluntary power of exhausting itself it to having eaten some bacon and beans for in individual cases. This and similar cases, supper the night before. I could not ascerwhich I can adduce, have made a great tain the nature of the evacuations; he had and lasting impression on me; they have not suffered any cramp or pain, and had led me on to a series of reflections, in which made water two hours previously; the exit were most unwise at this time to indulge, tremities were colder than natural, and of a and they have stamped indelibly on my blueish hue; the pulse was 60 in the mimind the gross absurdity of that precipi- nute; the countenance was lengthened, and tancy which crowds into the stomach a host characteristic of the disease. Great ad. of most heterogeneous medicines with dend- vantage having been speedily obtained $\mathrm{Jy}$ and empirical velocity. It is not my from the abstraction of blood in other cases wish to observe upon some other methods, which I had attended presenting the above otherwise than that, however great may be appearances, I was induced to employ their merits, regarded as improvements in bleeding in the present case, and with mechanism, they have added nothing to therapeutics. I bave the honour to be, Sir, Your most obedient servant,

Eoward Hickman,

Late Assistant-Surgeon H.C.'s Bengal Establishment.

P.S. Since writing the above, 1 have been particular in my inquiries respecting the habits of life and occupation of Jane Fayrer. The result is highly satisfactory. She takes in a little washing for one and always the same family. I have not been able to trace out either infection or contagion. She has lived in her present house for four-and-twenty years. Her husband has worked for Mr. Burrows, confectioner in Houndsditch, between twenty-two and three years. 'They are truly industrious and respectable persons. She is now quite recovered.

\title{
MALIGNANT CHOLERA
}

TREATED WTTII CARBONATE OF AMMONIA, SALINE DRAUGHTS, AND CALOMEL.

\section{To the Editor of The LANCET.}

Sir,-I beg leave to transmit to you the following accounts of two cases of cholera, in which the employment of carbonate of anmonia, with effervescing saline draughts frequently repeated, was very successful in the collapsed stage of the disease. I attended them conjointly with Mr. Rutherford, to whom I express my acknowledgments for the hints and unremitting cooperation afforded by him upon each occa. sion. I am, Sir,

Your obedient humble servant,

H. J. RadchifF, M.R.C.S.

Old Brentford, Sept. 18, 1832. difficulty obtained eight ounces of dark grumous blood, during which time the patient experienced severe cramps in the calves of his legs, had a rice-water stool, and was also seized with romiting, so that he immediately rejected the pills of calomel, camphor, and opium, which were given him. As it was evident that no reaction would be produced by the bleeding, but, on the contrary, that collapse was fast ap. proaching, strong brandy-and-water was given frequently, but always rejected by vomiting; frictions of the extremities wera also perseveringly used, but without any good effect, and at seven p.m. the pulse at the wrist became imperceptible, as also did the action of the heart. With the view of allaying the sickness, and administering a diffusible stimulus, I suggested to Mr. Rutherford the probable benefit which might result from an effervescing saline draught, containing five grains of carbonate of ammonia in excess; this the patient swallowed with avidity, and it completely allayed the vomiting, so that he retained the two before-mentioned pills which were given along with the draught. Sinapisms were applied to the sules of the feet, calves of the leys, and over the cardiac and epigastric regions; frictions were continued along the thighs and upper extremities, and $\mathbf{a}$ turpentine embrocation along the spine; the effervescing draughts, each containing three grains and a half of carbonate of ammonia in excess, were given every hour; at ten p. m. the pulse was still imperceptible, so that the draughts were continued every hour for three hours longer, as well as the frictions, by which time (one a.m. on the 21st) reaction commenced; and at half-past six a.m. the pulse had risen to 90 , and the heat of surface was equably restored, so that the stimulus had been discontinued since one o'clock. At eight $\mathbf{a . m}_{8}$, 
five grains of calomel and two of camphor temples, completely removing the cerebral were given, as slight cramps were felt in the excitement, and reducing the frequency of lower extremities, and the stools continued the pulse. The simple effervescing salines untinged with bile, but less frequent. Sim- were continued every two hours, and five ple effervescing draughts were given every grains of calomel were given at bèd-timé, two hours, and grain doses of calomel when no pain or cramp remained, and the every hour, until the secretions resumed heat of the surface wis equable over the their bilious character; the urine was body. The purging had also ceased since plentifully secreted on the 21 st. The con- noon. In the night urine was plentifully secutive fever, which was rather high, was secreted, and on the morning of the 23rd treated upon general principles, and in six it was found necessary to administer a dose days the patient's health was re-established, of castor oil, which procured a copious with the exception of weakness, and the bilious evacuation, after which the secuinconvenience produced by the blistered surfaces, which being very extensive were proportionably long in healing. This patient took 22 grains of calomel only, and 25 grains of carbonate of ammonia. It was on account of the patient's excessive thirst that the effervescing salines were exbibited so frequently, and also in consequence of his great partiality to them.

CASE 2.-W. E., æetat. four years and two months, was seized at midnight, on the 21st of August, with purging and vomiting of a fluid resembling rice-water, which continued very frequent until three a. $m$. on the 22nd, when 1 first saw him in a state of collapse, and suffering trom cramps in the extremities and stomach, over which I directed a sinapism to be applied, as well as others to the soles of the feet, and frictions to be incessantly used to the extremities. I ordered strong brandy -and-water to be given occasionally, and a powder immediately consisting of four grains of ealomel and half a grain of camphor, which being soon rejected by vomiting, was repeated. When I next visited this little patient at seven a.m., and found that the sickriess contirued, and the pulse was still imperceptible at the wrist and carotid arteries, I obtained Mr. Rutherford's sanction for administering an effervescing draught containing two grains of carbonate of ammonia in excess, which completely allayed the vomiting, and enabled him to retain another powder containing five grains of calomel, half a grain of camphor, and a quarter of a grain of opium, as the crimps continued, and we were doubtful whether all the previous dose of calomel and camphor had been retained. As the sinapisms also had not acted, they were renewed, the frictions continued, and an effervescing draught administered every half hour (each draught containing two grains of carbonate of ammonia in excess), until thirteen doses, or twenty-six grains of carbonate of ammonia, had been taken, when reaction speedily took place to more than the desired extent, as the pulse soon rose to 120 in the minute, and the head became affected, so that the stimulus was of course instantly discontinued, and leeches were applied to the tive fever alone remained to be treated, by mild aperients, diaphoretics, and the effervescing salines. On the 25th the patient was convalescent.

ALKALINE REMEDIES IN

\section{NALIGNANT CHOLERA.}

\section{To the Editor of TuE Lancer.}

SIR, - Since the cholera broke out in England, statements of various cases have appeared weekly in T'ue Lascet, treated by the most opposite means with nearly equal success ; so that the young practitioner is so bewildered on encountering the disease, as hardly to know what remedy to employ, but in most instances he is impressed witli the idea, that he has to combat a most formidable disorder, and of course chooses the most powerful medicines and doses for the purpose. The croton oil, calomel, opium with brandy, and other stimulants employed liberally, form the materia medica on most occasions. My object is not to detract from the merits of the above remedies, but to give the result of about 100 cases successfully treated on a principle laid down previously to the employment of medicines, for without some notion of the cause of the disease, it is impossible to have a clear conception of the effect of remedial agents.

The cause of the disease, I have not the least hesitation in asserting, from repeated experiments, to be a superabundance of acidity in the prime viæ, produced by the effect of infectious air, or by the combined operation of other substances.

Having a*certained the cause, the appropriate remedies will be obvious. At the commencement of the disease, for the purpose of correcting acidity, as well as of removing crudites that may be lodged in the stomach, I invariably give

$$
\begin{aligned}
& \text { Pulv. rheri; } \\
& \text { Mag.calimat.a. Эj. M. }
\end{aligned}
$$

Afterwards, two tablespoonfuls of the following mixture after every motion, or the same quantity every two hours, in case 